\title{
Fractal Analysis of the Material Surface Layer
}

\author{
Marina Katanaeva $^{1}$, Tatyana Lartseva ${ }^{1}$, Sergey Yudaev $^{1}$, Oleg Bavykin ${ }^{1}$, Olga Tambovtseva ${ }^{2}$ \\ ${ }^{1}$ Moscow Polytech University, Moscow, Russia \\ ${ }^{2}$ National Research University Higher School of Economics, Moscow, Russia
}

\begin{abstract}
To determine the surface quality of the machine parts, traditional methods based on the direct measurements of the microcracks are used. The results do not allow assessing the correlation of the surface layer quality and the given values. The approach developed recently measures the parameters of the surface layer using fractal analysis and solves this problem. To define the fractal dimension of the surface layer the modern digital stationary surface analyzers were used. As a result, it is possible to save the profile as a text file. This file is a time series consisting of the roughness heights of the surface elements. Having such a two-dimensional profile diagram, it is possible to calculate the fractal dimension in a computer program. This program will calculate the uploaded time series. Nowadays mathematicians use Fractan for time series fractal analysis. The results of the precision analysis of the data showed that Fractan has low precision in estimations of the time series fractal parameters. So Fractan cannot be used for the fractal analysis of the machine parts surface layer. The simultaneous use of the stationary surface analyzer and a computer program to estimate the Hurst exponent in order to define fractal characteristics of the material surface layer is proposed. Fractal analysis using the computer program Fractan shows low accuracy. A computer program written in VBA to calculate fractals of the time series is developed. The algorithms used in the program estimate the Hurst exponent with high accuracy.
\end{abstract}

Key words: Fractal analysis, material surface layer, quality, mathematical calculation, fractan computer program.

\section{INTRODUCTION}

There are different analysis and quality control methods, the most popular method recently has become the approach to study objects using fractal theory [1-5].

Authors use time series taken from the values of the material surface roughness heights as fractals.

It is known that the surface of the machine parts treated with modern methods has a difficult geometric shape. To determine the surface quality of the machine parts traditional methods based on the direct measurements of the microcracks are used. The results do not allow to assess the correlation of the surface layer quality and the given values. The approach developed recently measures the settings of the surface stratum using fractal analysis (based on the value of the fractal dimension - parameter D or based on the value of the Hurst exponent - parameter $\mathrm{H}$ ) and solves this problem.

Currently two key features of surface roughness formation can be distinguished:

- the roughness is seen not as a result of the cutting instrument, but as a quality of the structure itself;

- while processing the material with modern methods there appear elements on the surface. Their shape does not coincide with traditional roughness concept, where roughness is seen as a total of depressions and projections.

Taking everything into account, the traditional step by step and height parameters do not allow to estimate the roughness of the material adequately.

So new numerical characteristics of roughness are being developed. One of these characteristics is fractal dimension.

The correlation of the surface layer characteristics and its fractal dimension has been estimated $[1,2,6]$ :

- the correlation of the fractal dimension and the tribotechnical parameters (coefficient of friction) of the diamond-like material;

- the correlation of the parameter D of the polymer surface layer and the dynamic module elasticity and resilience;

- the correlation of the fractal dimension of tungsten and the speed of the surface etching in the system of water mono-ethanolamine - sodium chloride;

- the interrelation of the thickness of the polymer produced in the glow discharge of the diminished pressure and the fractal dimension;

- the correlation of the durability of samples made of martensitic steel and Parameter D.

There is a hypothesis that for machine parts with a complex form fractal dimension (Parameter D) can be used to estimate the surface layer roughness and the working properties of the parts.

Nowadays there are the technical methods to calculate Parameter D:

- a device for radio-optical calculations (Fig. 1) [6];

- scanning probe microscopy (SPM) [7-10]. 


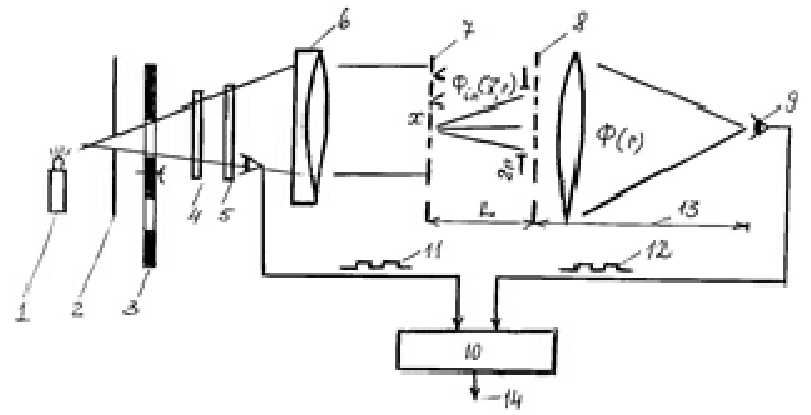

Figure 1: A device for radio-optical measurements

The device for radio-optical calculations is a prototype and is used only to analyze land. It does not allow to make fractal analysis of the material without introducing changes into the device and the operating modes.

SPM are highly accurate measuring instruments. The computer programs used to work with SPM can calculate parameter $\mathrm{D}$ based on the scanning of the specimen. However, such microscopes are very expensive, they can analyze only the specimen with certain linear dimensions, small scanning area of the surface and the necessity of rigid measuring conditions (in some cases the experiments are conducted only in vacuum). These criteria confine the use of scanning probe microscopes to scientific research only.

Yakovlev, Milovzorov [11] describe the idea of the traditional high roughness parameters calculation based on the use of a flatbed scanner and a PC program to process the scanned images. This approach has some disadvantages, such as inaccuracies of the roughness estimation due to the loss of some information while scanning and the transformation of the colour zones into numbers of the roughness heights.

To define the fractal dimension of the surface layer the modern digital stationary surface analyzers were used. As a result, it is possible not only to get the surface profile, but to save the profile as a text file. This file is a time series consisting of the roughness heights of the surface elements. Having such a two-dimensional profile diagram it is possible to calculate the fractal dimension in a computer program. This computer program will calculate the uploaded time series

\section{MATERIALS AND METHODS}

As was mentioned above, fractal analysis implies the calculation of fractal characteristics. As a rule, the Hurst exponent (parameter $\mathrm{H}$ ) or the fractal dimension value (parameter D) is estimated. To estimate parameter H, Hurst method is used (method of the standardized range, R/S analysis [1, 2]. This method is based on the following formula:

$$
\frac{\pi}{5}=(a r)^{H}
$$

$\alpha$-index (according to the classical method, $\alpha=1$, some authors suggest different values, such as $\alpha=0.5$ or $\alpha=1.57$ ); $\tau$ - dimension (range value);

$\mathrm{H}$ - Hurst exponent value (from 0 to 1 );

$\mathrm{R}$ - peak amplitude deviation of the time series;

$\mathrm{S}$ - standard deviation of the time series.

The value of $\mathrm{H}$ is estimated in two ways:

1) the exact way: finding the tangent of the gradient angel of the function $\mathrm{R} / \mathrm{S}$ from $\tau$, made in log-log scale;

2) approximate way: calculation by the given formula; Hurst exponent is estimated for the last element in the time series.

Using $\mathrm{H}$ parameter the time series can be estimated as follows :

- time series shows fractal qualities if $\mathrm{H}=0 \ldots 0.5$ and $\mathrm{H}=$ $0.5 \ldots 1$

- time series is described with the help of Markov process if $\mathrm{H}=0,5$; and by a non-Markov process if $\mathrm{H}=0 \ldots 0.5$ and $\mathrm{H}=0.5 \ldots 1$.

Furthermore, Hurst exponent can help estimate the correlation of the "past" time series and the "future" time series: there is a persistent correlation when $\mathrm{H}=0,5 \ldots 1$; there is an antipersistent correlation when $\mathrm{H}=0 \ldots 0,5$

The meaning of the fractal dimension of the surface layer is calculated using the formula (Eq. 2):

$$
D=3-H
$$

Fig. 2-4 show time series with different values of $\mathrm{H}$ exponent.

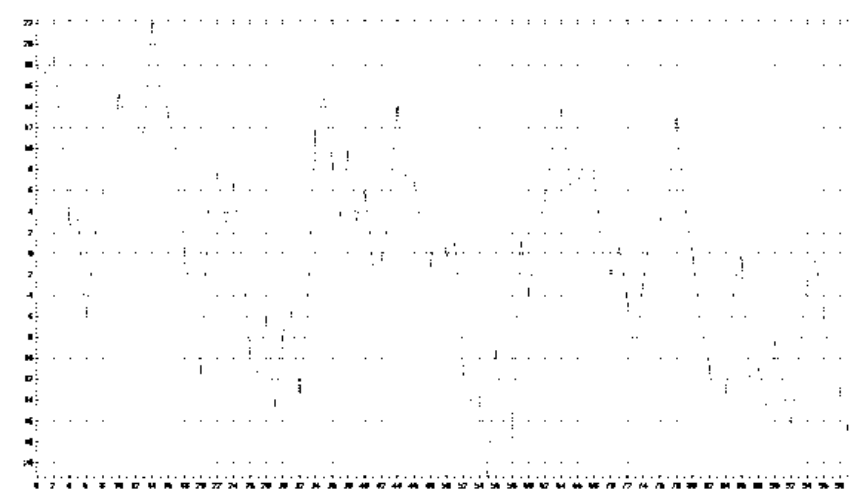

Figure 2: Time series with the parameter $\mathrm{H}=0.20$

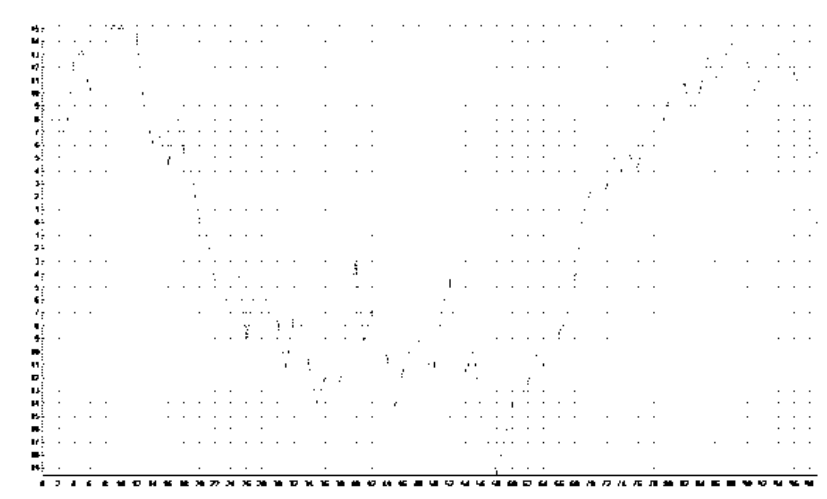

Figure 3: Time series with the parameter $\mathrm{H}=0$ 


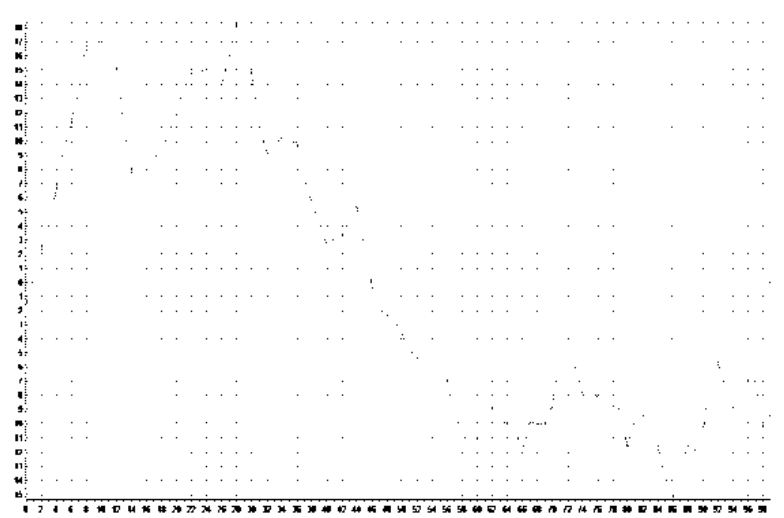

Figure 4: Time series with the parameterH=0.90

The researchers in fractal analysis sphere use Fractan [12-14]. The program is used to do mathematical simulation and to process data mathematically. The program enables researches to estimate Hurst exponent via the calculation of tangent of the tilting angle of the function $\mathrm{R} / \mathrm{S}$ of $\tau$ (the calculations use the classical value $\alpha=1$ ).

Fractan is very popular due to several reasons:

- it is a freeware program,

- the program contains algorithms that create Henon attractors, Lorenz attractors, Russell attractors, algorithms that generate time series with definite set parameters;

- the program can estimate the fractal dimension of the time series;
- the program has English and Russin interface;

- the program operates with high speed.

There is no information about the accuracy of the algorithms of Fractan, so it is not possible to choose the program for fractal analysis of the machine parts surface layer.

To define the accuracy of the algorithm of Fractan fractal analysis the following methodology was developed. There are the following steps:

1. Start Fractan and create a time series with set parameters: the length of the time series, mean-square deviation, the value of $\mathrm{H}$ parameter.

2. Save the created time series in ASCII format. Each file consists of one column that has the value of the time series. After creating the text file, close Fractan.

3. Open Fractan one more time. Open text file generated during Step 2, then process it: estimate $\mathrm{H}$ parameter for the whole time series.

4. Repeat steps 1-3 for other time series with different parameters.

Tables 1-2 show the initial data and the results of the fractal processing of time series in Fractan. The time frame generated in all experiments was 10000 elements long (according to Feder [1], it is appropriate to conduct $\mathrm{R} / \mathrm{S}$-analysis if the time series is longer than 2500 elements).

Table 1: The initial data and results of the fractal process of the time series of the generalized Brownian noise in Brownian motion in Fractan

\begin{tabular}{|c|c|c|c|c|}
\hline Generalized series & \multicolumn{3}{|c|}{ Characteristics of the created series } & Processing of the time series \\
\hline \multirow{4}{*}{ Generalized Brownian noise } & № & Hurst exponent & Average standard deviation & Estimated Hurst exponent \\
\cline { 2 - 5 } & 1 & 0.5 & 10 & 0.4432 \\
\cline { 2 - 5 } & 2 & 0.2 & 10 & 0.2144 \\
\cline { 2 - 5 } & 3 & 0.8 & 10 & 0.9015 \\
\hline \multirow{2}{*}{$\begin{array}{c}\text { Generalized Brownian } \\
\text { motion }\end{array}$} & 4 & 0.5 & 10 & 0.9634 \\
\cline { 2 - 5 } & 5 & 0.2 & 10 & 0.9998 \\
\cline { 2 - 5 } & 6 & 0.8 & 10 & 1.0125 \\
\hline
\end{tabular}

Table 2: The initial data and results of the fractal process of the Gaussian noise time series of in Fractan

\begin{tabular}{|c|c|c|c|}
\hline Characteristic of the produced Gaussian & \multicolumn{2}{|c|}{$\begin{array}{c}\text { Hurst exponent estimated in Fractan } \\
\text { noise }\end{array}$} & $\begin{array}{c}\text { Theoretical value of the } \\
\text { Hurst exponent } H\end{array}$ \\
\cline { 1 - 2 } № & $\begin{array}{c}\text { Average standard } \\
\text { deviation }\end{array}$ & 0.54230 & \multirow{2}{*}{0.5000} \\
\hline 7 & 25 & 0.47570 & \\
\hline 8 & 100 & 0.37280 & \\
\hline 9 & 500 & 0.66410 & \\
\hline 10 & 1000 & 0.66330 & \\
\hline 12 & 10 & 0.42650 & \\
\hline
\end{tabular}

The analysis of the obtained results shows:

a) Hurst exponent for time series of the generalized 
Brownian noise is estimated with high precision;

b) Hurst exponent for the generalized Brownian motion differs from the set parameters and is about 1,0. Moreover, estimated values of parameter $\mathrm{H}$ for time series 6 exceed the maximum theoretical value, which equals 1.

\section{RESULTS}

Having analyzed the results of the precision of Fractan fractal algorithm it is possible to suggest that there are reasons for deviations between theoretical and practical data. The reasons can be

- mistakes in the algorithm of the modelling of time series;

- mistakes in the algorithm for the Hurst exponent estimation;

- the influence of $\alpha$ index used in R/S analysis;

- the overall impact of any two or all reasons mentioned.

To estimate the fractal indexes of the time series more precisely a new computer program can be written.

This software should have more functions than Fractan, such as:

- Hurst exponent estimation using different $\alpha$ indices;

- $\mathrm{H}$ parameter estimation with high precision and approximately.

It is possible to create such software with the help of Excel or Calc.

To estimate parameter $\mathrm{H}$ it is necessary to enter the numbers of the series values in column «A».

Then the user needs to press a virtual button on the Excel spreadsheet (Fig. 5). Then there opens a pop-up window (Fig. 6 ) with the area for entering parameter $\alpha$.

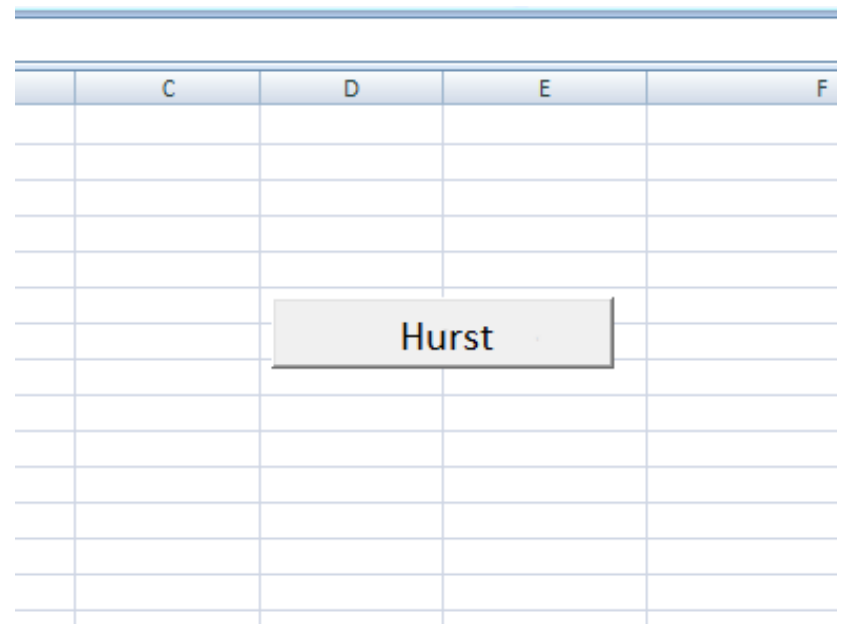

Figure 5: Launch of the R/S-analysis

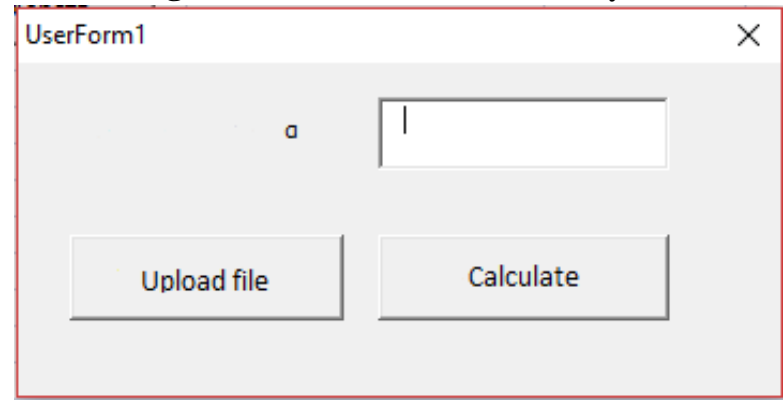

Figure 6: A window for entering the $\alpha$ index.

After a command there display a special window with the calculations of the parameter $\mathrm{H}$ and parametr D (diagram 6)

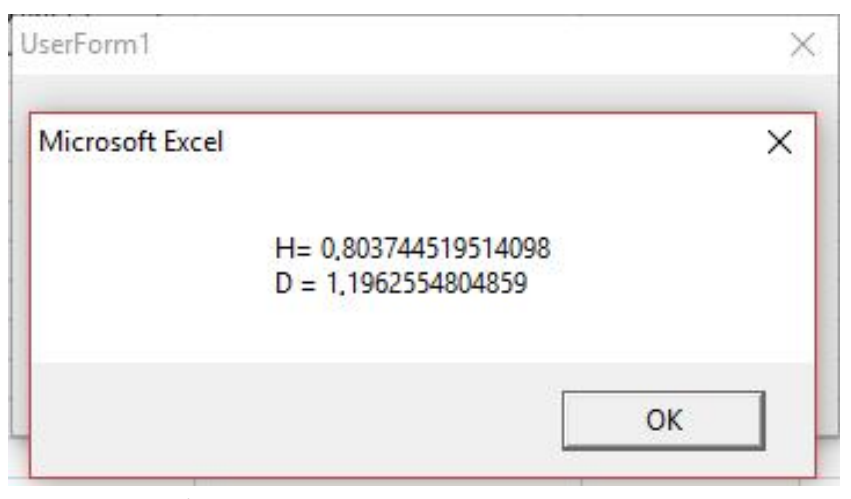

Figure 7: Fractal indexes calculation

\section{DISCUSSION}

To estimate the precision of the fractal information processing in the new computer program the following methods were introduced:

1) Fractan creates a time series with the specified feed data: the lenght of the time series, the average standard deviation, Hurst parameter $\mathrm{H}=0.5$.

2) The given time series is saved into a text file in DAT format. The file consists of the column with the values of the time series.

3) The researcher needs to open Excel and to upload the time series there.

4) The Hurst exponent is estimated.

5) Steps 1-4 are repeated for a time series with different parameters.

Table 3 shows the results of the time series fractal analysis using Fractan and the new software.

Table 3: The comparative results of the time series fractal analysis using Fractan and the new software

\begin{tabular}{|l|l|l|l|}
\hline \multicolumn{2}{|c|}{ Characteristics of the produced Gaussian noise } & Hurst exponent estimated in Fractan & $\begin{array}{l}\text { Hurst exponent estimated with } \\
\text { the new software }\end{array}$ \\
\cline { 1 - 2 } № & Average standard deviation & &
\end{tabular}


Marina Katanaeva et al., International Journal of Emerging Trends in Engineering Research, 8(8), August 2020, 4378 - 4382

\begin{tabular}{|l|l|l|l|}
\hline 7 & 25 & 0.5423 & 0.51845 \\
\hline 8 & 100 & 0.4757 & 0.50273 \\
\hline 9 & 500 & 0.3728 & 0.49946 \\
\hline 10 & 1000 & 0.6641 & 0.51374 \\
\hline 11 & 10 & 0.6633 & 0.50236 \\
\hline 12 & 5 & 0.4265 & 0.51003 \\
\hline
\end{tabular}

The table shows that the new software analyzes fractals with more precision: the Hurst exponent is closer to the theoretical value compared to the numbers Fractan shows (theoretical value for the Gaussian noise is $\mathrm{H}=0.5$ ).

\section{CONCLUSION}

1. Fractal analysis enables scientists to research not only the dynamic systems but to evaluate the functional properties of the machine parts and quality of the machine parts surface layer

2. To calculate fractal parameters of the material surface layer the best approach is to use the complex of modern stationary surface analyzers and a computer program for the $\mathrm{R} / \mathrm{S}$-analysis of the time series.

3. Nowadays mathematicians use Fractan for time series fractal analysis. The results of the precision analysis of the data showed that Fractan has low precision in estimations of the time series fractal parameters. So Fractan cannot be used for the fractal analysis of the machine parts surface layer.

4. A new software program has been written to conduct the time series fractal analysis. The program is based on Excel and is written in VBA. This program does the fractal calculations with more precision: the Hurst exponent indices are closer to the theoretical ones. By contrast, Fractan shows less precise results.

\section{REFERENCES}

[1] J. Feder. Fractals. New York: PlenumPub. Corp, 1988.

[2] A.A. Potapov, A.V. Laktyun'kin. Frequency coherence function of a space-time radar channel forming images of an anisotropic fractal surface and fractal objects. J. of Communications Technology and Electronics, no. 9, pp. 962-969, 2015.

[3] K.V. Malyshev, A.A. Potapov, S.L. Chernyshov. Figurate superlattices in the classical problem of signal filtering against the noise background on the basis of a cellular nonlinear network. $J$. of Communications Technology and Electronics, no. 4, pp. 325-332, 2013.

[4] E.S. Zhvansky, S.I. Pekov, A.A. Sorokin, V.A. Eliferov, I.A. Popov, V.A. Shurkhay, A.A. Potapov, E.N. Nikolaev. Metrics for evaluating the stability and reproducibility of mass spectra. J. Scientific Reports, vol. 9, p. 914, 2019.

[5] A.A. Gavdush, N.V. Chernomyrdin, K.M. Malakhov, G.R. Musina, O.P. Cherkasova, G.A. Komandin, K.I. Zaytsev, I.N. Dolganova, G.M. Katyba, V.E. Karasik,
S.I. Beshplav, A.V. Kosyrkova, P.V. Nikitin, A.A. Potapov, I.V. Reshetov, V.V. Tuchin. Terahertz spectroscopy of gelatin-embedded human brain gliomas of different grades: a road toward intraoperative THZ diagnosis. J.l of Biomedical Optic, vol. 24, no. 2, pp. 1-5, 2019.

https://doi.org/10.1117/1.JB0.24.2.027001

[6] O.B. Bavykin. Application in education of specialized computer programs "NOVA" and "MYTESTX". J.of IDO Science, no. 1, pp. 10-11, 2011.

[7] E.Y. Chaplygin, R.A. Morozov, V.K. Nevolin. On the possibility of biopolymer fragment analysis by tunneling microscopy. J. of Biophysics, vol. 1, no. 60, pp. 25-29, 2015.

[8] M.S. Ryabykh, E.S. Soynikova, V.M. Mikhelev. Development of information system for analysis of results of spm microscopy of blood cells. Electronic scientific journal, no. 6, pp. 40-43, 2017.

[9] I.L. Heglund, E.M. Kristyuk. Development of a program for calibration of the probe microscope piezoscanner based on measurements of TGS1 substrates. Science of the present and future, pp. 652-655, 2018.

[10]P.V. Gulyaev, E.Yu. Shelkovnikov, A.V. Tyrikov. Hardware-software for measurement of probe microscope sample movements. J. Measurement technology, no. 10, pp. 24-27, 2018. https://doi.org/10.32446/0368-1025it.2018-10-24-27

[11] A.V. Yakovlev, A.N. Milovzorov. Evaluation of results in the system of automated analysis of surface roughness. International collection of scientific articles MI VlGU5, no. 5, pp. 42-44, 2001.

[12]Sh.A. Mirzakulova, G.M. Yusupova, D.A. Nurpeisova. Time series analysis in fractan program. $J$. Eurasian Scientific Association, no. 48, pp. 66-69, 2019.

[13] Sh.A. Mirzakulova, L.O. Balgabekova. Research of multiservice network traffic in the program "FRACTAN", J. Science and business: ways of development, no. 9, pp. 78-82, 2013.

[14] N.V. Kireeva, M.A. Buranova. Exploring self-similar traffic using the fractan package. J. T-Comm: telecommunications and transport, no. 5, pp. 50-52, 2012. 